\title{
Fragility Index of block tailed vectors
}

\author{
Helena Ferreira Department of Mathematics, University of Beira Interior, Covilhã, Portugal \\ Marta Ferreira Department of Mathematics, University of Minho, Braga, Portugal
}

\begin{abstract}
Financial crises are a recurrent phenomenon with important effects on the real economy. The financial system is inherently fragile and it is therefore of great importance to be able to measure and characterize its systemic stability. Multivariate extreme value theory provide us such a framework through the fragility index (Geluk [10, et al., 2007; Falk and Tichy, 6, 7] 2010, 2011). Here we generalize this concept and contribute to the modeling of the stability of a stochastic system divided into blocks. We will find several relations with well-known tail dependence measures in literature, which will provide us immediate estimators. We end with an application to financial data.
\end{abstract}

Keywords: multivariate extreme value theory, tail dependence, fragility index, extremal coefficients

\section{Introduction}

In the last decade, dependencies between financial asset returns have increased, mostly as a consequence of globalization effects and relaxed market regulation. Therefore, the concept of tail dependence has been discussed in financial applications related to market or credit risk, e.g., Hauksson et al. [12] (2001), Ané and Kharoubi [1] (2003), Junker and May [16] (2005), Embrechts and Puccetti [5] (2010). The natural framework to model extremal dependence turns out to be the multivariate extreme value theory. The study of systemic stability is an important issue within this context of extreme risk dependence. The fragility of a system have been addressed to the Fragility Index (FI) introduced in Geluk et al. ([10, 2006). More precisely, consider a random vector $\mathbf{X}=\left(X_{1}, \ldots, X_{d}\right)$ and $N_{x}:=\sum_{i=1}^{d} \mathbb{1}_{\left\{X_{i}>x\right\}}$ the number of exceedances among $X_{1}, \ldots, X_{d}$ above a threshold $x$. The FI corresponding to $\mathbf{X}$ is the asymptotic conditional expected number of exceedances, given that there is at least one exceedance, i.e., $F I=\lim _{x \rightarrow \infty} E\left(N_{x} \mid N_{x}>0\right)$. The stochastic system $\left\{X_{1}, \ldots, X_{d}\right\}$ is called fragile whenever $F I>1$. Theoretical developments, namely, the asymptotic distribution of $N_{x}$ conditional to $N_{x}>0$ can be seen in Falk and Tichy (6. 17, 2010, 2011).

In this work we generalize some properties of the FI presented in the references above, contributing to the modeling of the stability of a stochastic system divided into blocks.

We shall state some notation that will be used throughout the paper.

Consider $\mathcal{D}=\left\{I_{1}, \ldots, I_{s}\right\}$ a partition of $D=\{1, \ldots, d\}$. For the random vector $\mathbf{X}=\left(X_{1}, \ldots, X_{d}\right)$, let $\mathbf{X}_{I_{j}}$ be a sub-vector of $\mathbf{X}$ whose components have indexes in $I_{j}$, with $j=1, \ldots, s$. If $F$ denotes the d.f. of $\mathbf{X}$ then $F_{I_{j}}$ denotes the d.f. of sub-vector $\mathbf{X}_{I_{j}}, j=1, \ldots, s$, and $F_{i}$ the marginal d.f., $i=1, \ldots, d$. Let $\mathbf{x}_{I_{j}}$ be a vector of length $\left|I_{j}\right|$ with components equal to $x \in \mathbb{R}$. We will say that $\mathbf{X}_{I_{j}}$ is the $j^{\text {th }}$ block of random vector $\mathbf{X}$ and denote by $N_{\mathbf{x}}$ the number of blocks where it occurs at least one exceedance of $\mathbf{x}$, i.e.,

$$
N_{\mathbf{x}}=\sum_{j=1}^{s} \mathbb{1}_{\left\{\mathbf{X}_{I_{j}} \not \mathbf{x}_{I_{j}}\right\}} .
$$

All operations and inequalities on vectors are meant componentwise.

Definition 1.1. The Fragility Index (FI) of a random vector $\mathbf{X}=\left(X_{1}, \ldots, X_{d}\right)$ relative to partition $\mathcal{D}$ is

$$
\lim _{x \rightarrow \infty} E\left(N_{\mathbf{x}} \mid N_{\mathbf{x}}>0\right)
$$


whenever the limit exists and is denoted $F I(\mathbf{X}, \mathcal{D})$.

If we consider $I_{j}=\{j\}, j=1, \ldots, d$, we find the FI introduced in Geluk et al. ([10, 2007) and latter study by Falk and Tichy (6, 7], 2010, 2011). This partition will be denoted as $\mathcal{D}^{*}$, i.e., $\mathcal{D}^{*}=\left\{I_{j}=\{j\}: j=1, \ldots, d\right\}$.

We give particular emphasis to random vectors in the domain of attraction of a multivariate extreme value distribution (MEV) and consider either the case of identically distributed margins or tail equivalent margins in the sense considered in Falk and Tichy ([7, 2011). In Section 2 we present some asymptotic properties of the distribution of $N_{x}$ conditional to $N_{x}>0$, and find generalizations of results in Falk and Tichy ([7], 2011). We prove that $F I(\mathbf{X}, \mathcal{D})$ exists and relates with the extremal coefficients $\epsilon$ of Tiago de Oliveira (24], 1962/63) and Smith (23], 1990) in case of identically distributed margins (Section 3). We define generalized versions of the multivariate tail dependence coefficients of $\mathrm{Li}([20,2009)$ and extend some of its results (Section 4). In Section 5 we relate these latter coefficients with $F I(\mathbf{X}, \mathcal{D})$.

For independent margins we have an unit FI. However, the stability of a stochastic system at higher levels can also be characterized by asymptotic independence (Geluk et al., [10, 2007). Asymptotic independence means that the dependency when present vanishes at extreme quantiles and the system is said to be weakly fragile, albeit possibly correlated (e.g., gaussian vectors). We extend the concept of asymptotic independent FI in Geluk et al. ([10, 2007) for blocks. A second measure is also presented by extending the 2-blocks asymptotic independent coefficient in Ferreira and Ferreira $(8], 2011)$ to the case of $s$-blocks, with $s>2$. This issue is considered in Section 6 .

Our results relating $F I(\mathbf{X}, \mathcal{D})$ with well-known tail dependence measures, for which estimators and respective properties have already been study in literature, will provide us immediate estimators (Section 7). We end with an application to financial data.

\section{Asymptotic Properties of $N_{x}$}

In this section we present some asymptotic properties of the distribution of $N_{x}$ conditional to $N_{x}>0$. We start to relate this latter with $\epsilon_{A}^{G}$, the extremal coefficients (Tiago de Oliveira, 24] 1962/63; Smith, 23] 1990) of the sub-distribution functions of the MEV $G$ corresponding to margins in $A$, i.e., by assuming $G$ has unit Fréchet margins,

$$
\epsilon_{A}^{G}=-\log G\left(\mathbb{1}_{A}^{-1}(1), \ldots, \mathbb{1}_{A}^{-1}(d)\right)
$$

where, for all $A \subset D, x \in \mathbb{R}$,

$$
\mathbb{1}_{A}^{-1}(x)= \begin{cases}1 & , x \in A \\ \infty & , x \notin A .\end{cases}
$$

They may be written through the stable tail dependence function (Huang, [14] 1992):

$$
l_{G}\left(x_{1}^{-1}, \ldots, x_{d}^{-1}\right)=-\log G\left(x_{1}, \ldots, x_{d}\right)=-\log C_{G}\left(e^{-1 / x_{1}}, \ldots, e^{-1 / x_{d}}\right),
$$

where $C_{G}$ is the copula of $G$, i.e.,

$$
G\left(x_{1}, \ldots, x_{d}\right)=C_{G}\left(G_{1}\left(x_{1}\right), \ldots, G_{d}\left(x_{d}\right)\right), \quad\left(x_{1}, \ldots, x_{d}\right) \in \mathbb{R}^{d} .
$$

In the sequel we will use notation $\mathcal{I}(A)=\cup_{j \in A} I_{j}$.

Proposition 2.1. If $\mathbf{X}$ has d.f. F with identically distributed continuous margins and belongs to the domain of attraction of a $M E V G$ with unit Fréchet margins then, for each $k \in\{1, \ldots, s\}$, we have

$$
\lim _{x \rightarrow \infty} P\left(N_{x}=k \mid N_{x}>0\right)=\frac{1}{\epsilon_{D}^{G}} \sum_{S \subset\{1, \ldots, s\} ;|S|=k} \sum_{T \subset S}(-1)^{|T|+1} \epsilon_{\mathcal{I}\left(T \cup S^{C}\right)}^{G}
$$


Dem. We have, successively,

$$
\begin{aligned}
P\left(N_{x}=k \mid N_{x}>0\right) & =\frac{1}{1-P\left(N_{x}=0\right)} \sum_{S \subset\{1, \ldots, s\} ;|S|=k} P\left(\cap_{j \in S} \mathbf{X}_{I_{j}} \not \mathbf{x}_{I_{j}}, \cap_{j \notin S} \mathbf{X}_{I_{j}} \leq \mathbf{x}_{I_{j}}\right) \\
& =\frac{1}{1-P\left(N_{x}=0\right)} \sum_{S \subset\{1, \ldots, s\} ;|S|=k} \sum_{T \subset S}(-1)^{|T|} P\left(\cap_{j \in T \cup S^{C}} \mathbf{X}_{I_{j}} \leq \mathbf{x}_{I_{j}}\right) \\
& =\frac{1}{1-F(\mathbf{x})} \sum_{S \subset\{1, \ldots, s\} ;|S|=k} \sum_{T \subset S}(-1)^{|T|+1}\left(1-F\left(x \mathbb{1}_{\mathcal{I}\left(T \cup S^{c}\right)}^{-1}(1), \ldots, x \mathbb{1}_{\mathcal{I}\left(T \cup S^{c}\right)}^{-1}(d)\right)\right)
\end{aligned}
$$

since $\sum_{T \subset S}(-1)^{|T|+1}=0$. Assuming w.l.o.g. that $F$ has unit Pareto marginals, we obtained

$$
\begin{aligned}
& P\left(N_{x}=k \mid N_{x}>0\right)= \\
= & \frac{1}{1-C_{F}\left(\mathbf{1}-\frac{1}{x} \mathbf{1}\right)} \sum_{S \subset\{1, \ldots, s\} ;|S|=k} \sum_{T \subset S}(-1)^{|T|+1}\left(1-C_{F}\left(1-\frac{1}{x} \mathbb{1}_{\mathcal{I}\left(T \cup S^{c}\right)}^{-1}(1), \ldots, 1-\frac{1}{x} \mathbb{1}_{\mathcal{I}\left(T \cup S^{c}\right)}^{-1}(d)\right)\right) .
\end{aligned}
$$

By hypothesis, $F$ belongs to the domain of attraction of a $\mathrm{MEV} G$, which is equivalent to (de Haan and de Ronde, 11 1998):

$$
\lim _{t \rightarrow \infty} \frac{1-C_{F}\left(1-y_{1} / x, \ldots, 1-y_{d} / x\right)}{1 / x}=-\log C_{G}\left(e^{-y_{1}}, \ldots, e^{-y_{d}}\right),\left(y_{1}, \ldots, y_{d}\right) \geq \mathbf{0}
$$

Taking limits in (5) and dividing both members by $1 / x$, conditions (6) and (7) lead us to

$$
\begin{aligned}
& \lim _{x \rightarrow \infty} P\left(N_{x}=k \mid N_{x}>0\right)= \\
= & \frac{1}{-\log C_{G}\left(e^{-1}, \ldots, e^{-1}\right)} \sum_{S \subset\{1, \ldots, s\} ;|S|=k} \sum_{T \subset S}(-1)^{|T|+1}\left(-\log C_{G}\left(e^{-\mathbb{1}_{\mathcal{I}\left(T \cup S^{c}\right)}(1)}, \ldots, e^{-\mathbb{1}_{\mathcal{I}\left(T \cup S^{c}\right)}(d)}\right)\right) \\
= & \frac{1}{\epsilon_{D}^{G}} \sum_{S \subset\{1, \ldots, s\} ;|S|=k} \sum_{T \subset S}(-1)^{|T|+1} \epsilon_{\mathcal{I}\left(T \cup S^{C}\right)}^{G} .
\end{aligned}
$$

The previous result can be generalized to random vectors $\mathbf{X}$ with equivalent marginal distributions, in the sense that, there exists a d.f. $H$ such that,

$$
\lim _{x \rightarrow w(H)} \frac{1-F_{i}(x)}{1-H(x)}=\gamma_{i} \in(0, \infty), i=1, \ldots, d,
$$

where $w(H)$ is the right-end-point of $H$. In this case it is no longer possible an interpretation based on extremal coefficients, as can be seen in the following result.

Proposition 2.2. If $\mathbf{X}$ has d.f. $F$ with equivalent marginal distributions in the sense of (8), and belongs to the domain of attraction of a MEV $G$ with unit Fréchet margins then, for each $k \in$ $\{1, \ldots, s\}$, we have

$$
\begin{aligned}
& \lim _{x \rightarrow w(H)} P\left(N_{x}=k \mid N_{x}>0\right)= \\
= & \frac{1}{\log C_{G}\left(e^{-\gamma_{1}}, \ldots, e^{-\gamma_{d}}\right)} \sum_{S \subset\{1, \ldots, s\} ;|S|=k} \sum_{T \subset S}(-1)^{|T|+1} \log C_{G}\left(e^{-\gamma_{1} \mathbb{1}_{\mathcal{I}\left(T \cup S^{c}\right)}(1)}, \ldots, e^{-\gamma_{d} \mathbb{1}_{\mathcal{I}\left(T \cup S^{c}\right)}(d)}\right)
\end{aligned}
$$

Dem. Observe that

$$
\begin{aligned}
& 1-F\left(x \mathbb{1}_{\mathcal{I}\left(T \cup S^{c}\right)}^{-1}(1), \ldots, x \mathbb{1}_{\mathcal{I}\left(T \cup S^{c}\right)}^{-1}(d)\right) \\
= & 1-C_{F}\left(1-\left(1-F_{1}\left(x \mathbb{1}_{\mathcal{I}\left(T \cup S^{c}\right)}^{-1}(1)\right)\right), \ldots, 1-\left(1-F_{1}\left(x \mathbb{1}_{\mathcal{I}\left(T \cup S^{c}\right)}^{-1}(d)\right)\right)\right) \\
= & 1-C_{F}\left(1-t_{1}^{\mathcal{I}\left(T \cup S^{c}\right)}(x), \ldots, 1-t_{d}^{\mathcal{I}\left(T \cup S^{c}\right)}(x)\right)
\end{aligned}
$$

where

$$
t_{i}^{A}(x)=\mathbb{1}_{A}(i) \frac{1-F_{i}(x)}{1-H(x)}(1-H(x)), i=1, \ldots d .
$$


Applying (8), we have

$$
\begin{aligned}
& \lim _{x \rightarrow w(H)} \frac{1-C_{F}\left(1-t_{1}^{\mathcal{I}\left(T \cup S^{c}\right)}(x), \ldots, 1-t_{d}^{\mathcal{I}\left(T \cup S^{c}\right)}(x)\right)}{1-H(x)} \\
= & -\log C_{G}\left(e^{-\gamma_{1} \mathbb{1}_{\mathcal{I}\left(T \cup S^{c}\right)}(1)}, \ldots, e^{-\gamma_{d} \mathbb{1}_{\mathcal{I}\left(T \cup S^{c}\right)}(d)}\right) .
\end{aligned}
$$

The result follows by retaking expression in (5) and considering (9) and (10).

If in particular we consider $I_{j}=\{j\}, j=1, \ldots, d$, we find the result of Falk and Tichy $([7,2011)$.

The results above can also be obtained through the relation between $C_{G}$ and $D$-norms presented in Aulbach et al. (2], 2011). However, we have chosen to present self-contained proofs using the usual arguments of multivariate extreme value theory that are more familiar.

\section{The Fragility Index for blocks}

In this section we compute the FI for blocks given in (1), whenever $\mathbf{X}$ has equally distributed or tail equivalent margins in the sense of (8), belonging to the domain of attraction of a MEV $G$ with unit Fréchet margins.

Proposition 3.1. If $\mathbf{X}$ has d.f. F with identically distributed continuous margins and belongs to the domain of attraction of a MEV G with unit Fréchet margins, we have

$$
F I(\mathbf{X}, \mathcal{D})=\frac{\sum_{j=1}^{s} \epsilon_{I_{j}}^{G}}{\epsilon_{D}^{G}}
$$

Dem. Observe that

$$
\begin{aligned}
F I(\mathbf{X}, \mathcal{D}) & =\sum_{j=1}^{s} \lim _{x \rightarrow \infty} P\left(\mathbf{X}_{I_{j}} \not \mathbf{x}_{I_{j}} \mid N_{x}>0\right) \\
& =\sum_{j=1}^{s} \lim _{x \rightarrow \infty} \frac{1-F\left(x \mathbb{1}_{I_{j}}^{-1}(1), \ldots, x \mathbb{1}_{I_{j}}^{-1}(d)\right)}{1-F(x, \ldots, x)} \\
& =\sum_{j=1}^{s} \lim _{x \rightarrow \infty} \frac{1-C_{F}\left(1-\frac{1}{x} \mathbb{1}_{I_{j}}(1), \ldots, 1-\frac{1}{x} \mathbb{1}_{I_{j}}(d)\right) /(1 / x)}{1-C_{F}\left(1-\frac{1}{x}, \ldots, 1-\frac{1}{x}\right) /(1 / x)} \\
& =\frac{1}{\epsilon_{D}^{G}} \sum_{j=1}^{s} \epsilon_{I_{j}}^{G} .
\end{aligned}
$$

In particular, for partitions corresponding to the family of all margins we obtain the known result $F I\left(\mathbf{X}, \mathcal{D}^{*}\right)=d / \epsilon_{D}^{G}$. Hence, the FI of the system divided into blocks is smaller than the system itself, i.e., $F I(\mathbf{X}, \mathcal{D}) \leq F I\left(\mathbf{X}, \mathcal{D}^{*}\right)$.

Remark 3.1. We can relate $F I(\mathbf{X}, \mathcal{D})$ with the fragility indexes of the whole system and of each block. More precisely, $F I(\mathbf{X}, \mathcal{D})$ is a convex linear combination of the ratios $F I\left(\mathbf{X}, \mathcal{D}^{*}\right) / F I\left(\mathbf{X}_{I_{j}}, \mathcal{D}_{I_{j}}^{*}\right)$, since we can write

$$
F I(\mathbf{X}, \mathcal{D})=\sum_{j=1}^{s} \frac{\left|I_{j}\right|}{d} \frac{F I\left(\mathbf{X}, \mathcal{D}^{*}\right)}{F I\left(\mathbf{X}_{I_{j}}, \mathcal{D}^{*}\right)} .
$$

Furthermore, it is also a weighted mean of those ratios:

$$
F I(\mathbf{X}, \mathcal{D})=\frac{1}{s} \sum_{j=1}^{s} \frac{\left|I_{j}\right| s}{d} \frac{F I\left(\mathbf{X}, \mathcal{D}^{*}\right)}{F I\left(\mathbf{X}_{I_{j}}, \mathcal{D}^{*}\right)} .
$$

Proposition 3.2 (inter-blocks dependence). Under the conditions of Proposition 3.1, we have 
(i) $1 \leq F I(\mathbf{X}, \mathcal{D}) \leq \frac{\sum_{j=1}^{s} \epsilon_{I_{j}}^{G}}{\bigvee_{j=1}^{s} \epsilon_{I_{j}}^{G}}$

(ii) $F I(\mathbf{X}, \mathcal{D})=1$ if and only if $\mathbf{X}_{I_{j}}, j=1, \ldots s$ are independent random vectors.

(iii) $F I(\mathbf{X}, \mathcal{D})=\frac{\sum_{j=1}^{s} \epsilon_{I_{j}}^{G}}{\bigvee_{j=1}^{s} \epsilon_{I_{j}}^{G}}$ if and only if $\mathbf{X}_{I_{j}}, j=1, \ldots s$ are totally dependent random vectors in the sense $G(\mathbf{x})=\bigwedge_{j=1}^{s} G_{I_{j}}\left(\mathbf{x}_{I_{j}}\right)$.

Dem. By the inequalities

$$
\prod_{j=1}^{s} G_{I_{j}}\left(\mathbf{x}_{I_{j}}\right) \leq G(\mathbf{x}) \leq \bigwedge_{j=1}^{s} G_{I_{j}}\left(\mathbf{x}_{I_{j}}\right)
$$

we have, successively,

$$
\begin{gathered}
\left(G_{\{1\}}(x)\right)^{\sum_{j=1}^{s} \epsilon_{I_{j}}^{G}} \leq G_{\{1\}}^{\epsilon_{D}^{G}}(x) \leq\left(G_{\{1\}}(x)\right)^{\bigvee_{j=1}^{s} \epsilon_{I_{j}}^{G}} \\
\bigvee_{j=1}^{s} \epsilon_{I_{j}}^{G} \leq \epsilon_{D}^{G} \leq \sum_{j=1}^{s} \epsilon_{I_{j}}^{G} .
\end{gathered}
$$

Proposition 3.3 (intra-blocks dependence). Under the conditions of Proposition 3.1, we have

(i) $\frac{s}{\epsilon_{D}^{G}} \leq F I(\mathbf{X}, \mathcal{D}) \leq \frac{d}{\epsilon_{D}^{G}}$.

(ii) $F I(\mathbf{X}, \mathcal{D})=\frac{d}{\epsilon_{D}^{G}}$ if and only if the sub-vectors $\mathbf{X}_{I_{j}}, j=1, \ldots s$, only have independent r.v.'s.

(iii) $F I(\mathbf{X}, \mathcal{D})=\frac{s}{\epsilon_{D}^{G}}$ if and only if the sub-vectors $\mathbf{X}_{I_{j}}, j=1, \ldots s$, only have totally dependent random r.v.'s.

Dem. Just observe that $1 \leq \epsilon_{I_{j}} \leq\left|I_{j}\right|$, with the lower and upper bounds corresponding to, respectively, complete dependence and independence of r.v.'s within sub-vectors $\mathbf{X}_{I_{j}}, j=1, \ldots s$.

Next result presents an extremal coefficient for the amount of dependence between $\mathbf{Y}_{I_{j}}, j=$ $1, \ldots, s, \mathbf{Y} \sim G$, through the FI of $\mathbf{X} \sim F$ in the domain of attraction of the MEV $G$.

Proposition 3.4. Under the conditions of Proposition 3.1, we have

$$
G(\mathbf{x})=\left(G_{I_{1}}\left(\mathbf{x}_{I_{1}}\right), \ldots, G_{I_{s}}\left(\mathbf{x}_{I_{s}}\right)\right)^{1 / F I(\mathbf{X}, \mathcal{D})} .
$$

Dem. Observe that

$$
\begin{aligned}
G(\mathbf{x}) & =G_{1}(x)^{\epsilon_{D}^{G}}=\left(G_{1}(x)^{\sum_{j=1}^{s} \epsilon_{I_{j}}^{G}}\right)^{1 / F I(\mathbf{X}, \mathcal{D})} \\
& =\left(G_{1}(x)^{\epsilon_{I_{1}}^{G}} \ldots G_{1}(x)^{\epsilon_{I_{s}}^{G}}\right)^{1 / F I(\mathbf{X}, \mathcal{D})} \\
& =\left(G_{I_{1}}\left(\mathbf{x}_{I_{1}}\right), \ldots, G_{I_{s}}\left(\mathbf{x}_{I_{s}}\right)\right)^{1 / F I(\mathbf{X}, \mathcal{D})} \cdot \square
\end{aligned}
$$

If we consider partition $\mathcal{D}^{*}$ in the previous result, we obtain the known relation (Smith, 23] 1990),

$$
G(\mathbf{x})=\left(G_{1}(x)^{d}\right)^{1 / F I\left(\mathbf{X}, \mathcal{D}^{*}\right)}=\left(G_{1}(x)^{d}\right)^{\epsilon^{G} / d}=G_{1}(x)^{\epsilon^{G}} .
$$

Observe also that we can write $F I(\mathbf{Y}, \mathcal{D})$ instead of $F I(\mathbf{X}, \mathcal{D})$ with $\mathbf{X}$ in the domain of attraction of the MEV distribution of $\mathbf{Y}$ since $\mathbf{Y}$ belongs to the same domain of attraction. We finish this section with a generalization of Proposition 3.1 to the case of equivalent margins and two illustrative examples. 
Proposition 3.5. If $\mathbf{X}$ has d.f. $F$ with equivalent marginal distributions in the sense of (8), and belongs to the domain of attraction of a $M E V G$ with unit Fréchet margins then, for each $k \in$ $\{1, \ldots, s\}$, we have

$$
F I(\mathbf{X}, \mathcal{D})=\frac{\sum_{j=1}^{s} \log C_{G}\left(e^{-\gamma_{1} \mathbb{1}_{I_{j}}(1)}, \ldots, e^{-\gamma_{d} \mathbb{1}_{I_{j}}(d)}\right)}{\log C_{G}\left(e^{-\gamma_{1}}, \ldots, e^{-\gamma_{d}}\right)}
$$

Example 3.1. We consider a random vector $\mathbf{X}$ of Example 3.2 in Falk and Tichy ([6], 2010), i.e., having components $X_{i}=\sum_{k=1}^{m} \lambda_{i k} Y_{k}$, where $Y_{1}, \ldots, Y_{m}$ are independent r.v.'s with Pareto $(\alpha)$ distribution, $\alpha>0$, and $\lambda_{i j} \geq 0$ such that $\sum_{k=1}^{m} \lambda_{i k}^{\alpha}=1, i=1, \ldots, d$. Taking for $H$ any of the distributions of the margins of $\mathbf{X}$, the equivalence condition (8) holds with $\gamma_{i}=1, i=1, \ldots, d$. The distribution of $\mathbf{X}$ belongs to the domain of attraction of

$$
G(\mathbf{x})=\exp \left(-\sum_{k=1}^{m} \vee_{i=1}^{d}\left(\frac{\lambda_{i k}}{x_{i}}\right)^{\alpha}\right), \mathbf{x}>\mathbf{0}
$$

with Fréchet margins, $G_{i}(x)=\exp \left(-x^{-\alpha}\right)$. Hence, for $\mathbf{u}=\left(u_{1}, \ldots, u_{d}\right) \in(\mathbf{0}, \mathbf{1})$,

$$
C_{G}(\mathbf{u})=\exp \left(-\sum_{k=1}^{m} \vee_{i=1}^{d} \lambda_{i k}^{\alpha}\left(-\log u_{i}\right)\right) .
$$

By Proposition 3.5, we have

$$
F I\left(\mathbf{X}, \mathcal{D}^{*}\right)=\frac{\sum_{j=1}^{d} \sum_{k=1}^{m} \vee_{i=1}^{d} \lambda_{i k}^{\alpha} \mathbb{1}_{\{j\}}(i)}{\sum_{k=1}^{m} \vee_{i=1}^{d} \lambda_{i k}^{\alpha}}=\frac{\sum_{j=1}^{d} \sum_{k=1}^{m} \lambda_{j k}^{\alpha}}{\sum_{k=1}^{m} \vee_{i=1}^{d} \lambda_{i k}^{\alpha}}=\frac{d}{\sum_{k=1}^{m} \vee_{i=1}^{d} \lambda_{i k}^{\alpha}} .
$$

as obtained in Falk and Tichy ([6], 2010). For any partition $\mathcal{D}$, we have

$$
F I(\mathbf{X}, \mathcal{D})=\frac{\sum_{j=1}^{s} \sum_{k=1}^{m} \vee_{i=1}^{d} \lambda_{i k}^{\alpha} \mathbb{1}_{I_{j}}(i)}{\sum_{k=1}^{m} \vee_{i=1}^{d} \lambda_{i k}^{\alpha}}
$$

To illustrate, consider $d=3=m, \alpha=1$ and weights

$$
\begin{array}{lll}
\lambda_{11}=4 / 8, & \lambda_{12}=2 / 8, & \lambda_{13}=2 / 8, \\
\lambda_{21}=1 / 8, & \lambda_{22}=1 / 8, & \lambda_{23}=6 / 8, \\
\lambda_{31}=3 / 8, & \lambda_{32}=2 / 8, & \lambda_{33}=3 / 8 .
\end{array}
$$

We have

$$
F I\left(\mathbf{X}, \mathcal{D}^{*}\right)=\frac{3}{4 / 8+2 / 8+6 / 8}=\frac{24}{12}=2
$$

and, for $\mathcal{D}=\{\{1,2\},\{3\}\}$,

$$
F I(\mathbf{X}, \mathcal{D})=\frac{(4 / 8+2 / 8+6 / 8)+(3 / 8+2 / 8+3 / 8)}{4 / 8+2 / 8+6 / 8}=\frac{20}{12}<2 .
$$

Example 3.2. If $G$ has copula

$$
C_{G}\left(u_{1}, \ldots, u_{d}\right)=\exp \left(-\left(\sum_{i=1}^{d}\left(-\log u_{i}\right)^{1 / \alpha}\right)^{\alpha}\right), 0<\alpha \leq 1,
$$

(symmetric logistic model) then, for any partition $\mathcal{D}$, we have

$$
F I(\mathbf{X}, \mathcal{D})=\frac{\sum_{j=1}^{s}\left|I_{j}\right|^{\alpha}}{d^{\alpha}}=\sum_{j=1}^{s}\left(\frac{\left|I_{j}\right|}{d}\right)^{\alpha},
$$

and $F I\left(\mathbf{X}, \mathcal{D}^{*}\right)=d^{1-\alpha}$ as already stated in Geluk et al. (10], 2007). In the symmetric model the FI is only a function of the blocks size. If we consider the more general asymmetric logistic model, whose copula is given by

$$
C_{G}\left(u_{1}, \ldots, u_{d}\right)=\exp \left\{-\sum_{k=1}^{q}\left(\sum_{i=1}^{d}\left(-\beta_{k i} \log u_{i}\right)^{1 / \alpha_{k}}\right)^{\alpha_{k}}\right\}
$$

where $\beta_{k i}$ are non-negative constantes such that $\sum_{k=1}^{q} \beta_{k i}=1, i=1, \ldots, d, 0<\alpha_{k} \leq 1, k=1, \ldots, q$, we obtain

$$
F I(\mathbf{X}, \mathcal{D})=\frac{\sum_{j=1}^{s} \sum_{k=1}^{q}\left(\sum_{i \in I_{j}} \beta_{k i}^{1 / \alpha_{k}}\right)^{\alpha_{k}}}{\sum_{k=1}^{q}\left(\sum_{i=1}^{d} \beta_{k i}^{1 / \alpha_{k}}\right)^{\alpha_{k}}}
$$




\section{Tail dependence for blocks}

In the following we always consider that $\mathbf{X}$ has continuous marginal d.f.'s. Consider notation $M\left(I_{j}\right)=\bigvee_{i \in I_{j}} F_{i}\left(X_{i}\right), j \in D$.

Definition 4.1. The upper-tail dependence coefficients of $\mathbf{X}$ corresponding to partition $\mathcal{D}$ of $D$ are defined by, for each $S \subsetneq\{1, \ldots, s\}$,

$$
\tau_{S}^{F}=\lim _{u \uparrow 1} P\left(\bigcap_{j \notin S} M\left(I_{j}\right)>u \mid \bigcap_{j \in S} M\left(I_{j}\right)>u\right)
$$

when the limit exists.

If we consider partition $\mathcal{D}^{*}$, then $S \subsetneq\{1, \ldots, d\}$ and we find the definition of $\operatorname{Li}(20,2009)$. Further, the case $s=2$ lead us to definition of Ferreira and Ferreira $(8,2011)$.

Consider

$$
\lambda_{S}^{F}:=\lim _{u \uparrow 1} \frac{P\left(\bigcap_{j \in S} M\left(I_{j}\right)>u\right)}{1-u}
$$

for each $S \subsetneq\{1, \ldots, s\}$. Hence we can write

$$
\tau_{S}^{F}=\frac{\lambda_{\{1, \ldots, s\}}^{F}}{\lambda_{S}^{F}}
$$

Observe that $\lambda_{S}^{F}$ corresponds to the multivariate upper-tail dependence coefficient $\Lambda_{U}\left(\mathbf{1}_{S}\right)$ in Schmidt and Stadtmüller (21, 2006), where $\mathbf{1}_{S}$ denotes the unit vector with dimension $|S|$. In particular, for partition $\mathcal{D}^{*}, \lambda_{\{i, j\}}^{F}=\Lambda_{U}(1,1)$ corresponds to the well-known bivariate tail dependence concept (Sibuya, 22] 1960; Joe, [15] 1993).

Before we relate the FI with the tail dependence coefficients corresponding to a partition, we present in this section some extensions of the results in Li ([20, 2009).

Proposition 4.1. If $\mathbf{X}$ has $M E V$ distribution $G$ with standard Fréchet margins and spectral measure $W$ defined on the d-dimensional unit sphere $S_{d}$ then, for each $S \subsetneq\{1, \ldots, s\}$, we have

$$
\tau_{S}^{G}=\frac{\int_{S_{d}} \bigwedge_{j=1}^{s} \bigvee_{i \in I_{j}} w_{i} d W(\mathbf{w})}{\int_{S_{d}} \bigwedge_{j \in s} \bigvee_{i \in I_{j}} w_{i} d W(\mathbf{w})}
$$

Dem. From the spectral representation of $G$ we obtain, for $u$ sufficiently close to 1 ,

$$
\begin{aligned}
P\left(\bigcap_{j=1}^{s} M\left(I_{j}\right)>u\right) & =1-\sum_{\emptyset \neq S \subset\{1, \ldots, s\}}(-1)^{|S|+1} G\left(-\frac{\mathbb{1}_{\mathcal{I}(S)}^{-1}(1)}{\log u}, \ldots,-\frac{\mathbb{1}_{\mathcal{I}(S)}^{-1}(d)}{\log u}\right) \\
& \approx 1-\sum_{\emptyset \neq S \subset\{1, \ldots, s\}}(-1)^{|S|+1}\left(1+\log u \int_{S_{d}} \bigvee_{i \in \mathcal{I}(S)} w_{i} d W(\mathbf{w})\right) \\
& =1-\sum_{\emptyset \neq S \subset\{1, \ldots, s\}}(-1)^{|S|+1}\left(1-(-\log u) \int_{S_{d}} \bigvee_{j \in S} \bigvee_{i \in I_{j}} w_{i} d W(\mathbf{w})\right)
\end{aligned}
$$

Since

$$
\sum_{\emptyset \neq S \subset\{1, \ldots, s\}}(-1)^{|S|+1}=1
$$

and

$$
\sum_{\emptyset \neq S \subset\{1, \ldots, s\}}(-1)^{|S|+1} \bigvee_{j \in S} a_{j}=\bigwedge_{j \in\{1, \ldots, s\}} a_{j},
$$

expression in (15) becomes

$$
\left.P\left(\bigcap_{j=1}^{s} M\left(I_{j}\right)>u\right) \approx(-\log u) \int_{S_{d}} \bigwedge_{j=1}^{s} \bigvee_{i \in I_{j}} w_{i} d W(\mathbf{w})\right)
$$


Analogously, we obtain

$$
\left.P\left(\bigcap_{j \in S} M\left(I_{j}\right)>u\right) \approx(-\log u) \int_{S_{d}} \bigwedge_{j \in S} \bigvee_{i \in I_{j}} w_{i} d W(\mathbf{w})\right) .
$$

For the particular case $\mathcal{D}^{*}$, the previous result is the one found in $\mathrm{Li}([20,2009)$. Note also that the numerator of (14) can be expressed through extremal coefficients as follows:

$$
\begin{aligned}
& \sum_{\emptyset \neq S \subset\{1, \ldots, s\}}(-1)^{|S|+1} \int_{S_{d}} \bigvee_{j \in S} \bigvee_{i \in I_{j}} w_{i} d W(\mathbf{w}) \\
= & \sum_{\emptyset \neq S \subset\{1, \ldots, s\}}(-1)^{|S|+1} \epsilon_{\mathcal{I}(S)}^{G} \\
= & \epsilon_{I_{1}}^{G}+\ldots+\epsilon_{I_{s}}^{G}-\left(\epsilon_{I_{1} \cup I_{2}}^{G}+\epsilon_{I_{1} \cup I_{3}}^{G}+\ldots\right)-\ldots+(-1)^{|S|+1} \epsilon_{I_{1} \cup \ldots \cup I_{s}}^{G},
\end{aligned}
$$

a generalization of result (15) in Ferreira and Ferreira ([8, 2011) where $s=2$.

The next result highlights the connections between tail dependence and extremal coefficients.

Corollary 4.2. Under the conditions of Proposition 4.1, we have

$$
\begin{aligned}
& \text { (i) } \lambda_{S}^{G}=\sum_{\emptyset \neq T \subset S}(-1)^{|T|+1} \epsilon_{\mathcal{I}(T)}^{G} \\
& \text { (ii) } \tau_{S}^{G}=\frac{\sum_{\emptyset \neq T \subset\{1, \ldots, s\}}(-1)^{|T|+1} \epsilon_{\mathcal{I}(T)}^{G}}{\sum_{\emptyset \neq T \subset S}(-1)^{|T|+1} \epsilon_{\mathcal{I}(T)}^{G}} .
\end{aligned}
$$

We end this section with a generalization of Theorem 2.6 in Li ([20, 2009), by adapting the arguments to subsets of $D$ that correspond to unions of blocks in $\mathcal{D}$.

Proposition 4.3. If $F$ belongs to the domain of attraction of a MEV $G$ with unit Fréchet margins then, for any partition $\mathcal{D}$ and $\emptyset \neq S \subset\{1, \ldots, s\}$, the non-null upper-tail dependence coefficients $\tau_{S}^{F}$ are the same as the corresponding ones of $G$.

\section{$5 \quad$ Fragility Index and tail dependence for blocks}

In this section we shall see that the asymptotic d.f.'s of the conditional probability of $k$ exceedances between blocks, $I_{1}, \ldots, I_{s}$, can be derived through the tail dependence coefficients given in (12). More precisely, if the d.f. $F$ of $\mathbf{X}$ has tail dependence coefficients $\lambda_{S}^{F}$ corresponding to partition $\mathcal{D}$, we can obtain $\lim _{x \rightarrow \infty} P\left(N_{x}=k \mid N_{x}>0\right)$ from these latter. In particular, for $F$ in the domain of attraction of a MEV $G$, besides the representations presented in the previous results, we can write those limiting probabilities through tail dependence coefficients $\lambda_{S}^{G}$.

Proposition 5.1. Let $\mathbf{X}$ be a random vector with d.f. $F$ with continuous and identically distributed margins. Let $\mathcal{D}$ be a partition of $D$ for which the tail dependence coefficients $\lambda_{S}^{F}$ corresponding to $F$ exist for each $S \subset\{1, \ldots, s\}$. Then,

(i) for each $k \in\{1, \ldots, s\}$,

$$
\lim _{x \rightarrow \infty} P\left(N_{x}=k \mid N_{x}>0\right)=\frac{\sum_{S \subset\{1, \ldots, s\},|S|=k} \sum_{T \subset S^{C}}(-1)^{|T|} \lambda_{T \cup S}^{F}}{\sum_{k=1}^{s} \sum_{S \subset\{1, \ldots, s\},|S|=k} \sum_{T \subset S^{C}}(-1)^{|T|} \lambda_{T \cup S}^{F}},
$$

as long as the numerator is non-null.

(ii) if $F$ belongs to the domain of attraction of a $M E V G$ with unit Fréchet marginals, the limits in (i) exist and coincide for both distributions, $F$ and $G$. 
Dem. Just observe that

$$
\lim _{x \rightarrow \infty} P\left(N_{x}=k \mid N_{x}>0\right)=\lim _{u \uparrow 1} \frac{\sum_{S \subset\{1, \ldots, s\},|S|=k} \sum_{T \subset S^{C}}(-1)^{|T|} P\left(\bigcap_{T \cup S} M\left(I_{j}\right)>u\right)}{\sum_{k=1}^{s} \sum_{S \subset\{1, \ldots, s\},|S|=k} \sum_{T \subset S^{C}}(-1)^{|T|} P\left(\bigcap_{T \cup S} M\left(I_{j}\right)>u\right)} .
$$

Now divide both terms of the ratio by $1-u$.

Analogously, we obtain the FI through $\lambda_{S}^{F}$ or $\lambda_{S}^{G}$.

Proposition 5.2. Under the conditions of Proposition 5.1, we have

(i)

$$
F I(\mathbf{X}, \mathcal{D})=\frac{\sum_{j=1}^{s} \lambda_{\{j\}}^{F}}{\sum_{k=1}^{s} \sum_{S \subset\{1, \ldots, s\},|S|=k} \sum_{T \subset S^{C}}(-1)^{|T|} \lambda_{T \cup S}^{F}}
$$

as long as the numerator is non-null.

(ii) if $F$ belongs to the domain of attraction of a $M E V G$ with unit Fréchet marginals, FI $(\mathbf{X}, \mathcal{D})$ exists and the the expression in (i) coincides for both distributions, $F$ and $G$.

The statements in (ii) of the two propositions are consequences of Proposition 4.3

\section{Asymptotic Independence}

If $\mathbf{X}$ has independent margins $X_{i}, i=1, \ldots, d$, we have an unit FI. As Geluk et al. ([10, 2007) observed, in this case we might have an asymptotic independence characterized by a dependency that vanishes at extreme quantiles. Asymptotic independence means that the system is weakly fragile, albeit possibly correlated (e.g., gaussian vectors). Geluk et al. (10, 2007) have defined a fragility index for asymptotic independence (AIFI) given by

$$
\eta_{D}=\frac{1}{d} \lim _{x \rightarrow \infty} \frac{\sum_{i=1}^{d} \log P\left(X_{i}>x\right)}{\log P\left(X_{1}>x, \ldots, X_{d}>x\right)} .
$$

In case $d=2$ we find the Ledford and Tawn coefficient of asymptotic independence ([18, 19, 1996, 1997) and, if $d>2$, a multivariate extension of this latter (Ferreira and Ferreira, [9], 2012).

Here we consider an extension of the AIFI in (16) for blocks, in the same spirit of the FI in Proposition 3.1, i.e., by relating the AIFI within blocks with the AIFI of the whole vector.

Let $\eta_{A}$ be the AIFI of sub-vector $\mathbf{X}_{A}$ of $\mathbf{X}$, with $A \subset D$, i.e.,

$$
\eta_{A}=\frac{1}{|A|} \lim _{x \rightarrow \infty} \frac{\sum_{i \in A} \log P\left(X_{i}>x\right)}{\log P\left(\mathbf{X}_{A}>\mathbf{x}_{A}\right)} .
$$

Definition 6.1. Let $\mathbf{X}=\left(X_{1}, \ldots, X_{d}\right)$ be a random vector with $F I(\mathbf{X}, \mathcal{D})=1$. Then the AIFI of $\mathbf{X}=\left(X_{1}, \ldots, X_{d}\right)$ relative to partition $\mathcal{D}$ of $D$ is

$$
\frac{1}{s} \lim _{x \rightarrow \infty} \frac{\sum_{j=1}^{s} \log P\left(\mathbf{X}_{I_{j}}>\mathbf{x}_{I_{j}}\right)}{\log P\left(X_{1}>x, \ldots, X_{d}>x\right)}
$$

whenever the limit exists and is denoted $\eta(\mathbf{X}, \mathcal{D})$.

Proposition 6.1. Let $\mathbf{X}=\left(X_{1}, \ldots, X_{d}\right)$ be a random vector with $F I(\mathbf{X}, \mathcal{D})=1$. Assume that (16) holds and that (17) holds for all $I_{j} \in \mathcal{D}, j=1, \ldots, s$, with limit given by $\eta_{I_{j}}$, respectively. If $\mathbf{X}$ has identically distributed or equivalent margins in the sense of (8), then

$$
\eta(\mathbf{X}, \mathcal{D})=\eta_{D} \frac{1}{s} \sum_{j=1}^{s} \frac{1}{\eta_{I_{j}}}
$$


Dem. Observe that by (16) and (17), then

$$
\eta(\mathbf{X}, \mathcal{D})=\lim _{x \rightarrow \infty} \eta_{D} \frac{\frac{1}{s} \sum_{j=1}^{s} \frac{1}{\eta_{I_{j}}\left|I_{j}\right|} \sum_{i \in I_{j}} \log P\left(X_{i}>x\right)}{\frac{1}{d} \sum_{i=1}^{d} \log P\left(X_{i}>x\right)},
$$

and by (8), we have

$$
\eta(\mathbf{X}, \mathcal{D})=\lim _{x \rightarrow \infty} \eta_{D} \frac{\frac{1}{s} \sum_{j=1}^{s} \frac{1}{\eta_{I_{j}}}\left(\frac{1}{\left|I_{j}\right|} \sum_{i \in I_{j}} \log \gamma_{i}+\log (1-H(x))\right)}{\frac{1}{d} \sum_{i=1}^{d} \log \gamma_{i}+\log (1-H(x))} .
$$

In particular, we have $\eta\left(\mathbf{X}, \mathcal{D}^{*}\right)=\eta_{D}$ and hence, the AIFI of the system divided into blocks is larger than the AIFI of the system itself, i.e., $\eta(\mathbf{X}, \mathcal{D}) \geq \eta\left(\mathbf{X}, \mathcal{D}^{*}\right)$.

Remark 6.1. In the particular case of $F I\left(\mathbf{X}, \mathcal{D}^{*}\right)>1$ we have $\eta(\mathbf{X}, \mathcal{D})=1$.

Observe also that, by Proposition [6.1, we can relate $\eta(\mathbf{X}, \mathcal{D})$ with the fragility indexes of the whole system and of each block. More precisely, $\eta(\mathbf{X}, \mathcal{D})$ is the arithmetic mean of the ratios $\eta\left(\mathbf{X}, \mathcal{D}^{*}\right) / \eta\left(\mathbf{X}_{I_{j}}, \mathcal{D}^{*}\right)$.

Example 6.1. Consider $\mathbf{X}=\left(X_{1}, \ldots, X_{d}\right)$ a standard d-variate Gaussian random vector with d.f. $\Phi_{d}\left(\cdot ;\left(\Sigma_{i, j}\right)_{i, j \in D}\right)$ having positive definite correlation matrix $\left(\Sigma_{i, j}\right)_{i, j \in D}$. We have, for all $A \subset D$, $\eta_{A}^{-1}=\mathbf{1}_{A}\left(\Sigma_{i, j}\right)_{i, j \in A}^{-1} \mathbf{1}_{A}^{T}$, the sum of all elements of the sub-matrix $\left(\Sigma_{i, j}\right)_{i, j \in A}^{-1}$ (Geluk et al., [10], 200\%; Hua and Joe, [13], 2011). For illustration, consider dimension $d=4$, constant correlation $\rho$, and take $s=3$ with $I_{1}=\{1,2\}, I_{2}=\{3\}$ and $I_{3}=\{4\}$. We have $\eta_{D}=(3 \rho+1) / 4, \eta_{I_{1}}=(\rho+1) / 2$ and $\eta_{I_{2}}=\eta_{I_{3}}=1$. Hence

$$
\eta(\mathbf{X}, \mathcal{D})=\frac{1}{3} \frac{3 \rho+1}{4}\left(\frac{2}{\rho+1}+1+1\right)=\frac{(3 \rho+1)(\rho+2)}{6(\rho+1)} .
$$

In the sequel we consider positive/negative association of a random vector in the sense of Ledford and Tawn ([18, 19], 1996, 1997).

Proposition 6.2 (inter-blocks asymptotic independence). Under the conditions of Proposition 6.1, we have

(i) $\eta(\mathbf{X}, \mathcal{D}) \leq \frac{1}{s}$ in case of positive association between sub-vectors $\mathbf{X}_{I_{j}}, j=1, \ldots s$, and $\eta(\mathbf{X}, \mathcal{D}) \geq$ $\frac{1}{s}$ in case of negative association.

(ii) $\eta(\mathbf{X}, \mathcal{D}) \geq \frac{\wedge_{j=1}^{s} \eta_{I_{j}}}{s} \sum_{j=1}^{s} \frac{1}{\eta_{I_{j}}}$.

(iii) $\eta(\mathbf{X}, \mathcal{D})=\frac{1}{s}$ if and only if the sub-vectors $\mathbf{X}_{I_{j}}, j=1, \ldots s$, are independent.

(iv) $\eta(\mathbf{X}, \mathcal{D})=\frac{\wedge_{j=1}^{s} \eta_{I_{j}}}{s} \sum_{j=1}^{s} \frac{1}{\eta_{I_{j}}}$ if and only if the sub-vectors $\mathbf{X}_{I_{j}}, j=1, \ldots s$, are totally dependent.

Dem. Observe that, $P\left(X_{1}>x, \ldots, X_{d}>x\right) \leq \wedge_{j=1}^{s} P\left(\mathbf{X}_{I_{j}}>\mathbf{x}_{I_{j}}\right)$, with the upper bound corresponding to total dependence between sub-vectors $\mathbf{X}_{I_{j}}, j=1, \ldots s$. Under positive association we have $P\left(X_{1}>x, \ldots, X_{d}>x\right) \geq \prod_{j=1}^{s} P\left(\mathbf{X}_{I_{j}}>\mathbf{x}_{I_{j}}\right)$ and negative association otherwise, with the bounds corresponding to independence between sub-vectors $\mathbf{X}_{I_{j}}, j=1, \ldots s$.

Proposition 6.3 (intra-blocks asymptotic independence). Under the conditions of Proposition 6.1, we have

(i) $\eta(\mathbf{X}, \mathcal{D}) \leq \frac{\eta_{D} d}{s}$ in case the sub-vectors $\mathbf{X}_{I_{j}}, j=1, \ldots s$, only have positively associated r.v.'s and $\eta(\mathbf{X}, \mathcal{D}) \geq \frac{\eta_{D} d}{s}$ in case of negative association.

(ii) $\eta(\mathbf{X}, \mathcal{D}) \geq \eta_{D}$ 
(iii) $\eta(\mathbf{X}, \mathcal{D})=\frac{\eta_{D} d}{s}$ if and only if the sub-vectors $\mathbf{X}_{I_{j}}, j=1, \ldots s$, only have independent r.v.'s.

(iv) $\eta(\mathbf{X}, \mathcal{D})=\eta_{D}$ if and only if the sub-vectors $\mathbf{X}_{I_{j}}, j=1, \ldots s$, only have totally dependent random r.v.'s.

Dem. For each $j=1, \ldots s$, under positive association of the r.v.'s in $\mathbf{X}_{I_{j}}$ we have $P\left(\mathbf{X}_{I_{j}}>\right.$ $\left.\mathbf{x}_{I_{j}}\right) \geq \prod_{i \in I_{j}} P\left(X_{i}>x\right)$, and hence $1 / \eta_{I_{j}} \leq\left|I_{j}\right|$, with the upper bound corresponding to independence. For negative association we have $P\left(\mathbf{X}_{I_{j}}>\mathbf{x}_{I_{j}}\right) \leq \prod_{i \in I_{j}} P\left(X_{i}>x\right)$. Observe also that total dependence within each block means $P\left(\mathbf{X}_{I_{j}}>\mathbf{x}_{I_{j}}\right)=\wedge_{i \in I_{j}} \gamma_{i}(1-H(x))$.

As already mentioned, the definition of the AIFI in (18) measures the asymptotic independent fragility of a system divided into blocks (sub-vectors), by relating the asymptotic independent fragility within the blocks and in the whole system. If in (16) we generalize for blocks the concept of an exceedance of a r.v., $X_{i}>x$, through events $\mathbf{X}_{I_{j}} \not \leq \mathbf{x}_{I_{j}}$, we obtain another coefficient for asymptotic tail independence. In this way, we extend the coefficient of asymptotic tail independence that was considered in Ferreira and Ferreira $(8,2011)$ for the particular case of a partition $\mathcal{D}=\left\{I_{1}, I_{2}\right\}$ of $D=\{1, \ldots, d\}$.

Definition 6.2. Let $\mathbf{X}=\left(X_{1}, \ldots, X_{d}\right)$ be a random vector with $F I(\mathbf{X}, \mathcal{D})=1$. The coefficient of asymptotic independence of $\mathbf{X}=\left(X_{1}, \ldots, X_{d}\right)$ relative to partition $\mathcal{D}$ of $D$ is

$$
\lim _{x \rightarrow \infty} \frac{1}{s} \frac{\sum_{j=1}^{s} \log P\left(\mathbf{X}_{I_{j}} \not \leq \mathbf{x}_{I_{j}}\right)}{\log P\left(\bigcap_{j=1}^{s}\left\{\mathbf{X}_{I_{j}} \not \leq \mathbf{x}_{I_{j}}\right\}\right)},
$$

whenever the limit exists, and is denoted $\eta_{\left(I_{1}, \ldots, I_{s}\right)}$.

The following result is therefore an immediate extension of Proposition 2.4 in Ferreira and Ferreira ([8], 2011).

Proposition 6.4. Let $\mathbf{X}=\left(X_{1}, \ldots, X_{d}\right)$ be a random vector with $F I(\mathbf{X}, \mathcal{D})=1$. Assume that the limit in (20) exists and, for all $\emptyset \neq K_{j} \subset I_{j}, j=1, \ldots, s$, (17) holds for $\cup_{j=1}^{s} K_{j}$. Then

$$
\eta_{\left(I_{1}, \ldots, I_{s}\right)}=\max \left\{\eta_{\left\{i_{1}, \ldots, i_{s}\right\}}: i_{j} \in I_{j}, j=1, \ldots, s\right\} .
$$

Similar to $\eta(\mathbf{X}, \mathcal{D})$, coefficient $\eta_{\left(I_{1}, \ldots, I_{s}\right)}$ is also based on the coefficient of Ledford and Tawn or multivariate extensions of this latter.

In the example below, one can see that the asymptotic tail independent coefficients, $\eta(\mathbf{X}, \mathcal{D})$ and $\eta_{\left(I_{1}, \ldots, I_{s}\right)}$, are different.

Example 6.2. Consider $\left\{V_{i}\right\}_{i \geq 1}$ an i.i.d. sequence of unit Pareto r.v.'s. Let $\left(X_{1}, X_{2}, X_{3}\right)$ be a random vector such that, $X_{1}=\min \left(V_{1}, V_{2}\right), X_{2}=\min \left(V_{2}, V_{3}\right)$ and $X_{3}=\min \left(V_{3}, V_{4}\right)$. If $\mathcal{D}=$ $\left\{I_{1}, I_{2}\right\}$, with $I_{1}=\{1,2\}$ and $I_{2}=\{3\}$, we have $P\left(X_{i}>x\right)=x^{-2}, i=1,2,3, P\left(X_{1}>x, X_{2}>\right.$ $x)=P\left(X_{2}>x, X_{3}>x\right)=x^{-3}$ and $P\left(X_{1}>x, X_{3}>x\right)=P\left(X_{1}>x, X_{2}>x, X_{3}>x\right)=x^{-4}$. Therefore

$$
F I(\mathbf{X}, \mathcal{D})=\lim _{x \rightarrow \infty}=\frac{P\left(\bigcup_{i=1}^{2}\left\{X_{i}>x\right\}\right)+P\left(X_{3}>x\right)}{P\left(\bigcup_{i=1}^{3}\left\{X_{i}>x\right\}\right)}=\frac{2 x^{-2}-x^{-3}+x^{-2}}{3 x^{-2}-2 x^{-3}-x^{-4}+x^{-4}}=1,
$$

$\eta(\mathbf{X}, \mathcal{D})=\frac{1}{2} \lim _{x \rightarrow \infty} \frac{\sum_{j=1}^{2} \log P\left(\mathbf{X}_{I_{j}}>\mathbf{x}_{I_{j}}\right)}{\log P\left(X_{1}>x, X_{2}>x, X_{3}>x\right)}=\frac{1}{2} \lim _{x \rightarrow \infty} \frac{\log P\left(X_{1}>x, X_{2}>x\right)+P\left(X_{3}>x\right)}{\log P\left(X_{1}>x, X_{2}>x, X_{3}>x\right)}=\frac{1}{2} \frac{-3-2}{-4}=\frac{5}{8}$ and

$$
\begin{aligned}
& \eta_{\left(I_{1}, I_{2}\right)}=\frac{1}{2} \lim _{x \rightarrow \infty} \frac{\log P\left(\mathbf{X}_{I_{1}} \not \mathbf{x}_{I_{1}}\right)+\log P\left(\mathbf{X}_{I_{2}} \not \mathbf{x}_{I_{2}}\right)}{\log P\left(\mathbf{X}_{I_{1}} \not \mathbf{x}_{I_{1}}, \mathbf{X}_{I_{2}} \not \mathbf{x}_{I_{2}}\right)} \\
& =\frac{1}{2} \lim _{x \rightarrow \infty} \frac{\log \left(P\left(X_{1}>x\right)+P\left(X_{2}>x\right)-P\left(X_{1}>x, X_{2}>x\right)\right)+\log P\left(X_{3}>x\right)}{\log \left(P\left(X_{1}>x, X_{3}>x\right)+P\left(X_{2}>x, X_{3}>x\right)-P\left(X_{1}>x, X_{2}>x, X_{3}>x\right)\right)} \\
& =\frac{1}{2} \lim _{x \rightarrow \infty} \frac{\log \left(2 x^{-2}-x^{-3}\right)+\log x^{-2}}{\log \left(x^{-4}+x^{-3}-x^{-4}\right)}=2 / 3 .
\end{aligned}
$$


Observe that the same results are obtained if we apply Propositions 6.1 and 6.4, respectively. More precisely, we have

$$
\begin{gathered}
\eta_{D}=\eta_{\{1,2,3\}}=\frac{1}{3} \lim _{x \rightarrow \infty} \frac{\sum_{i=1}^{3} \log P\left(X_{i}>x\right)}{\log P\left(X_{1}>x, X_{2}>x, X_{3}>x\right)}=\frac{1}{3} \frac{3 \log x^{-2}}{\log x^{-4}}=\frac{1}{2}, \\
\eta_{\{1,3\}}=\frac{1}{2} \frac{2 \log x^{-2}}{\log x^{-4}}=\frac{1}{2} \quad \text { and } \quad \eta_{\{2,3\}}=\frac{1}{2} \frac{2 \log x^{-2}}{\log x^{-3}}=\frac{2}{3} .
\end{gathered}
$$

Obviously $\eta_{I_{2}}=1$ and

$$
\eta_{I_{1}}=\frac{1}{2} \lim _{x \rightarrow \infty} \frac{\sum_{i=1}^{2} \log P\left(X_{i}>x\right)}{\log P\left(X_{1}>x, X_{2}>x\right)}=\frac{1}{2} \frac{2 \log x^{-2}}{\log x^{-3}}=\frac{2}{3} .
$$

Hence, by Propositions 6.1 and 6.4, respectively,

$$
\eta(\mathbf{X}, \mathcal{D})=\frac{1}{2}\left(\frac{1}{2}(3 / 2+1)\right)=\frac{5}{8}
$$

and

$$
\eta_{\left(I_{1}, I_{2}\right)}=\max \left\{\eta_{\{1,3\}}, \eta_{\{2,3\}}\right\}=\max \{1 / 2,2 / 3\}=2 / 3
$$

\section{Estimation of the Fragility Index for blocks}

In the previous sections we have relate the FI for blocks with well-known tail dependence measures. This will allow to obtain immediate estimators for our index through the estimators of those coefficients that are already studied in literature.

Proposition 3.1 presents an estimation procedure for the FI based on the extremal coefficients of Tiago de Oliveira $([24,1962 / 63)$ and Smith $([23,1990)$ given in (2). Observe that they can be expressed through the stable tail dependence function, $l_{G}$, defined in (3). There are several references in literature on the estimation of the stable tail dependence function. In a parametric framework, a model for $l_{G}$ must be imposed. Non-parametric estimators are usually based on an arbitrarily chosen parameter, corresponding to the number of top order statistics to use in order to provide the best trade-off between bias and variance, which is not an easy task. For a survey, we refer Krajina (17, 2010) or Beirlant et al. ([3] 2004). The more recent work in Ferreira and Ferreira $($ [8, 2011) presents a simpler non-parametric estimator based on sample means, which we shall adopt here.

For $A \subset D$, denote $M(A)=\bigvee_{i \in A} F_{i}\left(X_{i}\right)$. Consider

$$
\widehat{\epsilon}_{D}^{G}=\frac{\overline{M(D)}}{1-\overline{M(D)}} \quad \text { and } \quad \widehat{\epsilon}_{I_{j}}^{G}=\frac{\overline{M\left(I_{j}\right)}}{1-\overline{M\left(I_{j}\right)}}
$$

where $\overline{M(A)}$ is the sample mean

$$
\overline{M(A)}=\frac{1}{n} \sum_{i=1}^{n} \bigvee_{j \in A} \widehat{F}_{j}\left(X_{j}^{(i)}\right)
$$

and $\widehat{F}_{j}, j \in A$, is the (modified) empirical d.f. of $F_{j}$,

$$
\widehat{F}_{j}(u)=\frac{1}{n+1} \sum_{k=1}^{n} \mathbb{1}_{\left\{X_{j}^{(k)} \leq u\right\}} .
$$


The denominator $n+1$ instead of $n$ in the ordinary empirical d.f. concerns estimation accuracy and other modifications can be used (see, for instance, Beirlant et al. 3] 2004). Based on Proposition 3.1. and for a partition $\mathcal{D}$ of $D$, we consider estimator

$$
\widehat{F I}(\mathbf{X}, \mathcal{D})=\frac{\sum_{j=1}^{s} \widehat{\epsilon}_{I_{j}}^{G}}{\widehat{\epsilon}_{D}^{G}}
$$

which is consistent given the consistency of estimators $\widehat{\epsilon}_{D}^{G}$ and $\widehat{\epsilon}_{I_{j}}^{G}$ already stated in Ferreira and Ferreira ([8], 2011).

By Proposition 5.2, we can also estimate $F I(\mathbf{X}, \mathcal{D})$ based on the tail dependence coefficients in (12). As already mentioned, these correspond to multivariate upper-tail dependence coefficients considered in Schmidt and Stadtmüller ([21], 2006), for which non-parametric estimators have been studied. We remark that these estimators are also based on a similar procedure as described above for the stable tail dependence function, i.e., it comprises the choice of a number of top order statistics to be used, not too large neither too small to avoid, respectively, large bias and large variance.

Remark 7.1. In case of asymptotic independence considered in Section $\mathbf{6}$, immediate estimators for the asymptotic independent coefficients, $\eta(\mathbf{X}, \mathcal{D})$ and $\eta_{\left(I_{1}, \ldots, I_{s}\right)}$, can be derived from the found relations with the Ledford and Tawn coefficient (or multivariate extensions), whose estimation has already been studied in literature (see, for instance, Draisma [4], 2004 or, for a survey, Beirlant et al., [3], 2004). In particular, the Ledford and Tawn coefficient can be estimated as the extreme value index of r.v. $\min \left(1 /\left(1-F_{1}\left(X_{1}\right)\right), 1 /\left(1-F_{2}\left(X_{2}\right)\right)\right)$ and, similarly, its multivariate versions in (17) can be estimated as the extreme value index of r.v. $\min _{i \in A}\left(1 /\left(1-F_{i}\left(X_{i}\right)\right)\right)$.

\subsection{An application to financial data}

We now illustrate the estimation of the FI for blocks through an application to data analyzed by Ferreira and Ferreira (8], 2011). The data are the series of negative log-returns of the closing values of the stock market indexes, CAC 40 (France), FTSE100 (UK), SMI (Swiss), XDAX (German), Dow Jones (USA), Nasdaq (USA), SP500 (USA), HSI (China), Nikkei (Japan). The period covered is January 1993 to March 2004. Since we do not have a sample of maximum values, we consider the monthly maximums in each market. We group the indexes in Europe (CAC 40, FTSE100, SMI, XDAX), USA (Dow Jones, Nasdaq) and Far East (HSI, Nikkei). The presence of dependence within these groups was already evidenced in Ferreira and Ferreira (8], 2011). We are interested in assessing the fragility within the system of the financial stock markets whenever grouped in the three big world markets referred: Europe, USA and Far East. To this end, we use estimator $\widehat{F I}(\mathbf{X}, \mathcal{D})$ in (24). In Table 1 are the obtained estimates, as well as, the estimates of the extremal coefficient $\left(\widehat{\epsilon}^{G}\right)$ within each group and in the whole system (we denote the whole system, i.e., the vector of all observations as "Global"). The estimates of the FI within each financial market group and in the whole system are also presented. One can see that the USA is the most fragile financial system with $\widehat{F I}=1.885948905$. Observe also that the FI of the whole system is almost twice the FI of the system divided into blocks.

\section{References}

[1] Ané T., Kharoubi, C. (2003). Dependence structure and risk measure. J. Bus. Econom. Statist. $76,411-438$.

[2] Aulbach, S., Bayer, V. and Falk, M. (2011). A multivariate piecing-together approach with an application to operational loss data. Bernoulli, in print. 
[3] Beirlant, J., Goegebeur, Y., Segers, J. e Teugels, J. (2004). Statistics of Extremes: Theory and Application. John Wiley.

[4] Draisma,G., Drees, H., Ferreira, A. and de Haan, L. (2004). Bivariate tail estimation: dependence in asymptotic independence. Bernoulli 10, 251-280.

[5] Embrechts, P., Puccetti, G. (2006). Bounds for Functions of Dependent Risks. Finance Stoch. 10(3), 341-352.

[6] Falk, M. and Tichy, D. (2010). Asymptotic conditional distribution of exceedance counts. Revised version submitted.

[7] Falk, M. and Tichy, D. (2011). Asymptotic conditional distribution of exceedance counts: fragility index with different margins.

[8] Ferreira, H., Ferreira M. (2011). Extremal dependence: some contributions. arXiv:1108.1972v1 [math.ST].

[9] Ferreira, M., Ferreira, H. (2012). Tail dependence between order statistics. JMVA 105(1), 176-192.

[10] Geluk, J.L., De Haan, L. and De Vries, C.G. (2007). Weak and strong financial fragility. Tinbergen Institute Discussion Paper, TI 2007-023/2.

[11] de Haan, L. and de Ronde, J. (1998). Sea and wind: multivariate extremes at work. Extremes $17-45$.

[12] Hauksson, H., Dacorogna, M., Domenig, T., Mueller, U. and Samorodnitsky, G. (2001). Multivariate Extremes, Aggregation and Risk Estimation, Quantitative Finance 1, 79-95.

[13] Hua, L., Joe, H. (2011). Tail order and intermediate tail dependence of multivariate copulas. J. Multivariate Anal. 102(10), 1454-1471.

[14] Huang, X. (1992). Statistics of Bivariate Extreme Values. Ph. D. thesis, Tinbergen Institute Research Series 22, Erasmus University Rotterdam.

[15] Joe, H. (1997). Multivariate Models and Dependence Concepts, Chapman \& Hall, London.

[16] Junker, M., May, A. (2005). Measurement of aggregate risk with copulas. Econom. J. 8, 428454.

[17] Krajina, A. (2010). An M-Estimator of Multivariate Tail Dependence. Tilburg: Tilburg University Press.

[18] Ledford, A. and Tawn, J. A. (1996). Statistics for near independence in multivariate extreme values. Biometrika, 83, 169-187.

\begin{tabular}{c|c|c||c} 
& $\widehat{\epsilon}^{G}$ & $\widehat{F I}$ & $\widehat{F I}(\mathbf{X}, \mathcal{D})$ \\
\hline Europe & 2.243980009 & 1.78254707 & 1.370940479 \\
USA & 1.590711176 & 1.885948905 & \\
Far East & 1.673156121 & 1.195345715 & \\
\hline Global & 4.017568517 & 2.240160924 &
\end{tabular}

Table 1: Estimates of the extremal coefficient $\left(\widehat{\epsilon}^{G}\right)$ within each block (Europe, USA, Far East) and in the whole system (Global) comprising the three blocks, as well as, estimates of the FI $(\widehat{F I})$ within each block, in the whole system and within the system divided into blocks $(\widehat{F I}(\mathbf{X}, \mathcal{D}))$. 
[19] Ledford, A. and Tawn, J. A. (1997). Modelling Dependence within joint tail regions, J. R. Stat. Soc. Ser. B Stat. Methodol. 59, 475-499.

[20] Li, H. (2009). Orthant tail dependence of multivariate extreme value distributions, J. Multivariate Anal., 100(1), 243-256.

[21] Schmidt, R., Stadtmüller, U. (2006). Nonparametric estimation of tail dependence, Scandinavian J. Statist. 33 307-335.

[22] Sibuya, M. (1960). Bivariate extreme statistics, Ann. Inst. Statist. Math. 11 195-210.

[23] Smith, R.L. (1990). Max-stable processes and spatial extremes. Preprint, Univ. North Carolina, USA.

[24] Tiago de Oliveira, J. (1962/63). Structure theory of bivariate extremes, extensions. Est. Mat., Estat. e Econ. 7, 165-195. 